\title{
Eosinophilic Esophagitis and Achalasia: Two Distinct Nosologic Entities or a Possible Etiopathogenic Association?
}

\author{
Dimitrios Schizas $^{\text {a }}$ Athanasios Syllaios $^{\text {a }}$ Michail Vailas $^{\text {a }}$ Maria Sotiropoulou $^{\mathrm{a}}$ \\ Tania Triantafyllou $^{b}$ Dimitrios Tsapralis $^{c}$ Ioannis S. Papanikolaou ${ }^{d}$ \\ Dimitrios Theodorou ${ }^{b}$
}

${ }^{a}$ First Department of Surgery, National and Kapodistrian University of Athens, Laikon General Hospital, Athens, Greece; ${ }^{b}$ First Propedeutic Department of Surgery, National and Kapodistrian University of Athens, Hippocration General Hospital, Athens, Greece; 'Surgical Department, General Hospital of lerapetra, lerapetra, Greece; dHepatogastroenterology Unit, Second Department of Internal Medicine and Research Institute, National and Kapodistrian University of Athens, Attikon University Hospital, Athens, Greece

\author{
Keywords \\ Eosinophilic esophagitis · Achalasia · Esophagus · \\ Eosinophilia · Pathogenesis · Management
}

\begin{abstract}
Background: Despite the fact that researchers have made significant progress in elucidating the pathophysiology of esophageal diseases, the understanding of esophageal motility alterations in patients with eosinophilic esophagitis (EoE) is in its infancy and current published medical literature remains rather scarce on this topic. A growing body of scientific data regarding associations between esophageal motor disorders, such as achalasia and EoE, exists nowadays. Summary: It seems that the association of EoE and achalasia does not constitute a cause and effect relationship, as it is not clear whether esophageal motility abnormalities are the result of EoE or vice versa. As such, there is no universally accepted treatment algorithm for patients presenting with both of these entities. Key Messages: The aim of this article is to review the existing data on achalasia-like motility disorders in patients with EoE, highlighting a possible association
\end{abstract}

karger@karger.com

(c) 2021 S. Karger AG, Basel

www.karger.com/ddi

Karger $\stackrel{2}{5}$ between these 2 esophageal disorders. Moreover, we seek to describe the clinical presentation in such cases, diagnostic modalities to be used, and current treatment strategies in patients suspected to suffer from both disorders.

(c) 2021 S. Karger AG, Base

\section{Introduction}

Eosinophilic esophagitis (EoE) is nowadays considered a chronic allergic-immune condition of the esophagus that was not recognized as a unique clinicopathological entity up until 1993 [1]. Since then, its frequency has significantly increased, with its prevalence reaching 50 100 cases $/ 100,000$ persons solely in the USA [1].

Although EoE mainly refers to people between 20 and 40 years old, all age-groups may be affected. EoE shows a male predominance, with a male-to-female ratio of $3: 1$ [2, 3]. The most recent definition of EoE applies to patients with specific symptoms such as dysphagia and food impaction who concurrently show marked eosinophilic inflammation in endoscopic esophageal specimens [histo- 
logically a peak count of 15 eosinophils per high-power field (hpf) of esophageal biopsy tissue] $[2,4,5]$.

Even though dysphagia is a common, initially presenting symptom in patients with EoE, its etiology remains unclear [2]. Anatomic abnormalities such as strictures can only be found in a small proportion of patients with EoE, raising the possibility that there may be an underlying esophageal motility disorder [2]. Published literature regarding associations between esophageal motility disorders and EoE is limited. Lately, a growing number of studies have been published trying to elucidate the exact pathophysiology of dysphagia in these patients $[1,3]$. Interestingly enough, esophageal motility abnormalities have been found in 25-75\% of EoE patients [3]. Not only hypercontractile disorders such as nutcracker esophagus, jackhammer esophagus, hypertensive lower esophageal sphincter (LES), and distal esophageal spasm but also hypocontractile disorders, such as absent contractility, ineffective esophageal motility, and hypotensive LES, have been reported in EoE patients [1-3]. Achalasia is an esophageal motility disorder of unknown origin in which neurons in the myenteric plexus of the esophagus are diminished or completely destroyed, resulting in impaired peristalsis and incomplete deglutitive relaxation of the LES $[1,6]$. Recent studies have reviewed patients suffering from achalasia showing also pronounced esophageal infiltration with eosinophils $[1,3]$. Official clinical guidelines and recommendations from different committees are continuously updated, but, currently, no consensus exists on patients with EoE combined with achalasia [4, 7]. The aim of this article is to review the existing data on achalasia-like motility disorders in patients with EoE and investigate the possible association between these 2 disorders; as well as to approach the specific clinical picture, diagnostic modalities, and treatment strategies in patients suspected to suffer from both disorders.

\section{Potential Etiopathogenesis of EOE and Achalasia}

Achalasia results from the loss of the esophageal intramural neurons that normally mediate peristalsis and deglutitive relaxation of the LES. The exact cause of this degeneration process remains unknown [8]. As far as pathophysiology is concerned, the loss of the inhibitory innervation of the esophagus can be due to either extrinsic or intrinsic causes [8]. Extrinsic causes may include central nervous system lesions involving the dorsal motor nucleus or the vagal nerve fibers, while intrinsic loss may be due to loss of the inhibitory ganglion cells in the my- enteric plexus. Genetic inheritance, viral infections, and autoimmune processes have been also implicated in the pathogenesis of achalasia [4]. Finally, cytotoxic proteins (such as eosinophil cationic protein [ECP]) released by degranulating eosinophils have been reported to play a pivotal role as a potential cause of achalasia by destroying esophageal intramural neurons [9].

The pathogenesis of EoE seems to be the result of a complex interaction between various factors such as genetic background, environmental causes, and antigenic stimuli [2]. Exposure to allergens triggers the antigen presentation and differentiation of T helper 2 cells secreting interleukins (IL-4, IL-5, and IL-13). These cytokines further overexpress other mediators such as chemokine ligand 26/eotaxin-3 and the upregulation of periostin in epithelial cells and fibroblasts [2]. Desmoglein, calpain, and filaggrin along with recruitment and stimulation of eosinophils lead to disruption of the epithelial barrier of the esophagus. Eosinophils may also release IL-9 affecting mast cells [2]. The pathophysiological mechanism also involves propagation of basophils and plasma cells rich in IgG4 which are found in the lamina propria [2]. Subepithelial fibrosis, mediated by transforming growth factor (TGF- $\beta$ ) along with IL-13, contributes to the remodeling of the lamina propria [2].

A variety of esophageal motility disorders, including achalasia, have been described in EoE patients, although the exact pathogenesis is unknown [1]. In EoE patients, eosinophils infiltrate all layers of the esophageal wall, including the submucosa, muscularis propria, and adventitia, although recent studies propose the possible existence of 2 distinct forms, the mucosal-predominant and the muscle-predominant one $[1,3]$. Esophageal motility abnormalities are most likely to accompany EoE with muscular involvement [3].

Recently, 3 possible mechanisms have been described in order to explain the association between achalasia and EoE [3]. The first one is based on the notion that motility abnormalities are primary (not caused by esophageal eosinophils). Thus, esophageal stasis of retained food material causes irritation of the mucosa, inducing secretion of chemokines and attracting eosinophils in the area of interest. As such, treatments that improve esophageal emptying could improve mucosal eosinophilia according to this theory [3]. The second potential mechanism supports that EoE causes achalasia-like motility abnormalities through the release of eosinophil products causing muscle contraction (e.g., leukotriene $\mathrm{D} 4$, prostaglandin F2 alpha, thromboxane B2) and muscle relaxation (e.g., IL-6, IL-13) [1,3]. Also, EoE may cause achalasia through 
the release of neuroactive eosinophil products (e.g., major basic protein $[\mathrm{MBP}])$, that disrupt peristalsis and interfere with LES relaxation, and through the release of profibrotic products (e.g., TGF- $\beta$, IL-13, IL-8, VEGF) that induce tissue remodeling. Treatments that reduce esophageal eosinophilia should therefore reverse motility abnormalities [1, 3]. Last but not least, a third possible mechanism lies on the basis that EoE might cause achalasia through the release of cytotoxic eosinophil secretory products (e.g., MBP, eosinophil peroxidase, ECP, eosinophil-derived neurotoxin) which destroy the esophageal intramural neurons that mediate peristalsis and LES relaxation $[1,3]$. Under these circumstances, treatments aiming at esophageal eosinophilia might prevent further neuronal damage, whereas the motility abnormalities due to existing neuronal loss may be irreversible $[1,3,10]$. Other less dominant theories regarding mechanisms by which eosinophilic infiltration in EoE may induce achalasia are based on the fact that coculturing of fibroblasts with eosinophils in EoE may result in increased contraction of the fibroblasts, resulting in abnormal motility [10]. Furthermore, inflammatory cytokines such as IL-1 and IL- 6 produced in EoE may inhibit acetylcholinesterase release, resulting in esophageal dysmotility, and also possible interactions between eosinophils and mast cells with the esophageal microenvironment may produce abnormal motility in EoE patients [10]. Finally, a recent study demonstrated that mast cells can cause esophageal muscle cells to proliferate and differentiate into a more contractile phenotype, and also that mediators released by degranulating mast cells such as tryptase and histamine can activate smooth muscle contraction pathways. Thus, activated mast cells in the esophageal muscularis propria might cause esophageal motility abnormalities, including the failure of LES relaxation which is typical of achalasia [11].

\section{Evidence Suggesting that EoE Causes Achalasia}

The first report of achalasia associated with "eosinophilic esophagitis" was published in 1978 by Landres et al. [12]. They described a patient with achalasia who had biopsies of esophageal muscularis propria showing "heavy eosinophilic infiltration." The authors attributed achalasia to an unusual variant of eosinophilic gastroenteritis [12].

Since T-lymphocyte subpopulations are involved in coordinating eosinophil influx into tissues during allergic responses (the pathogenesis of EoE), some studies have underlined this finding as a potential pathogenetic mechanism of achalasia [13-15]. In 1994, Goldblum et al. [13] revealed, in a study of esophagectomy specimens from 42 patients with end-stage achalasia, eosinophils and lymphocytes infiltrating the myenteric plexus in all cases, with eosinophilia involving the muscularis propria in 22 of them (52\%). In another study with 11 patients suffering from early achalasia, myenteric neuritis with T-lymphocyte-predominant inflammation was documented, with a mixture of both lymphocytes and eosinophils found in 6 cases (55\%). In this study, the esophageal muscle biopsy specimens were taken during Heller myotomy [14]. Furthermore, a study of 46 patients with achalasia or esophagogastric junction outlet obstruction revealed inflammatory infiltrates in 8 cases (17\%), 7 of which were comprised predominantly of $\mathrm{T}$ lymphocytes, while 1 was predominantly of eosinophils. Esophageal muscle biopsies were obtained in this study during Heller myotomy, as well [15]. The admixture of T lymphocytes and eosinophils found in achalasic esophageal muscle in these studies is noteworthy, although reversibility to medical therapy in these patients was not tested.

\section{Cause: Cytotoxic Secretory Products That Destroy Intramural Neurons}

In this subcategory, eosinophils infiltrating the esophageal muscle might degranulate and release toxic proteins that destroy esophageal neurons causing achalasia. Motility abnormalities due to extensive neuronal loss may be irreversible [3]. In 1989, Tottrup et al. [16] reported positive immunohistochemical staining for ECP in biopsies of esophageal muscularis propria taken during Heller myotomy, in all 9 patients with primary (idiopathic) achalasia. They proposed that degranulating eosinophils release cytotoxic proteins that destroy esophageal intramural neurons causing achalasia.

Moreover, another study, including 28 achalasia patients who had esophageal muscle biopsies taken during peroral endoscopic myotomy (POEM), found eosinophil MBP and eosinophil-derived neurotoxin present in 24 cases $(86 \%)$. The authors suggested that achalasia with eosinophil infiltration of esophageal muscle might represent a subtype of EoE [17]. Reversibility to medical therapy in those patients was also not tested.

Cause: Myoactive and Neuroactive Secretory Products Released by Eosinophils

In this subcategory, eosinophils infiltrating the esophageal muscle release myoactive and neuroactive secretory products causing achalasia. Motility abnormalities should 
Table 1. Studies revealing relationship between achalasia and EoE and possible etiopathogenesis

\begin{tabular}{|c|c|}
\hline Authors & Main finding-outcome \\
\hline \multicolumn{2}{|c|}{ Evidence suggesting that EoE causes achalasia } \\
\hline Sodikof et al. [15] & T lymphocytes and eosinophils in $17 \%$ of achalasia patients \\
\hline Goldblum et al. [14] & $\begin{array}{l}\text { T-lymphocyte-predominant inflammation in early achalasia patients, mixture of both lymphocytes and } \\
\text { eosinophils in } 55 \% \text { of them }\end{array}$ \\
\hline Goldblum et al. [13] & $\begin{array}{l}\text { Eosinophils and lymphocytes infiltrating the myenteric plexus in all end-stage achalasia patients. Eosin- } \\
\text { ophilia involving the muscularis propria in } 52 \% \text { of them }\end{array}$ \\
\hline Landres et al. [12] & $\begin{array}{l}\text { Achalasia was attributed to an unusual variant of eosinophilic gastroenteritis due to heavy eosinophilic } \\
\text { esophageal infiltration }\end{array}$ \\
\hline \multicolumn{2}{|c|}{ Cytotoxic secretory products that destroy intramural neurons } \\
\hline Jin et al. [17] & $\begin{array}{l}\text { MBP and EDN in } 86 \% \text { of achalasia patients - achalasia with eosinophil infiltration of esophageal muscle } \\
\text { might represent a subtype of EoE }\end{array}$ \\
\hline Tøttrup et al. [16] & $\begin{array}{l}\text { Positive immunohistochemical staining for ECP in biopsies of esophageal muscularis propria in achala- } \\
\text { sia patients - degranulating eosinophils release cytotoxic proteins that destroy esophageal intramural } \\
\text { neurons causing achalasia }\end{array}$ \\
\hline \multicolumn{2}{|c|}{ Myoactive and neuroactive secretory products released by eosinophils } \\
\hline Ghisa et al. [23] & Topical steroids initially alleviated symptoms in $62.5 \%$ of EoE and achalasia patients \\
\hline Mathur et al. [24] & $\begin{array}{l}\text { Patient with eosinophilic gastroenteritis and achalasia fully responded to oral prednisolone and botuli- } \\
\text { num toxin injection }\end{array}$ \\
\hline Surdea-Blaga et al. [25] & Patient with EoE and achalasia successfully treated with prednisone, PPIs, and budesonide \\
\hline Nakajima et al. [19] & Normalization of esophageal motility disturbances in EoE patients treated with steroids \\
\hline Nennstiel et al. [20] & $86 \%$ of EoE patients with motility abnormalities successfully treated with topical steroid therapy \\
\hline Savarino et al. [22] & Normalization of esophageal motility disturbances in EoE patients treated with steroids \\
\hline Hejazi et al. [18] & Normalization of esophageal motility disturbances in EoE patients treated with steroids \\
\hline Lucendo et al. [21] & $\begin{array}{l}\text { After successful treatment with fluticasone in EoE patients with abnormal peristalsis; all patients pre- } \\
\text { sented improvement in esophageal motor problems and dysphagia }\end{array}$ \\
\hline \multicolumn{2}{|c|}{ Evidence suggesting that achalasia causes EoE } \\
\hline \multicolumn{2}{|c|}{ Esophageal stasis inducing secretion of chemokines that attract eosinophils } \\
\hline Frieling et al. [27] & Patient with EoE and concurrent achalasia successfully treated after pneumatic dilatation of the cardia \\
\hline Mandaliya et al. [26] & In achalasia patients with EoE, EoE was resolved after achalasia treatment \\
\hline Cools-Lartigue et al. [28] & $\begin{array}{l}35 \% \text { of patients with combined achalasia and esophageal eosinophilia showed a decrease in esophageal } \\
\text { eosinophilia after achalasia treatment }\end{array}$ \\
\hline
\end{tabular}

EoE, eosinophilic esophagitis; MBP, major basic protein; ECP, eosinophil cationic protein; EDN, eosinophil-derived neurotoxin.

be reversible with treatments that reduce esophageal eosinophilia because those secretory products do not cause extant neuronal loss [1, 3].

Different studies have reported normalization of esophageal motility disturbances in EoE patients treated with steroids [18-20]. In HIMEOS study, 86\% of EoE patients with motility abnormalities were successfully treat- ed with topical steroid therapy [20]. In a prospective study, Lucendo et al. [21] reported manometric abnormalities in 12 EoE patients. Out of them, 9 had abnormal esophageal peristalsis and received treatment with fluticasone. An esophageal manometry was repeated in $7 \mathrm{pa}-$ tients after the treatment with fluticasone; in all $7 \mathrm{pa}$ tients, both esophageal motor problems and dysphagia 
improved. Moreover, 2 reports included patients with achalasia who exhibited a return of normal peristalsis and LES deglutitive relaxation on follow-up manometry, performed after steroid treatment had reduced their esophageal eosinophilia [18, 22]. Ghisa et al. [23] reported $8 \mathrm{EoE}$ patients with concomitant achalasia. In their study, a significant proportion of EoE patients had achalasia (7.3\%) or obstructive motor disorders (14.7\%). Proton-pump inhibitors (PPIs) or topical steroids alleviated symptoms initially in $62.5 \%$ of patients, nevertheless the majority ( 5 out of 8 patients), finally, required invasive therapy to treat achalasia [23]. Mathur A. et al. [24] reported a patient with eosinophilic gastroenteritis presenting with achalasia that fully responded to oral prednisolone and botulinum toxin injection both clinically and manometrically. In another report, the authors successfully treated a patient with EoE and achalasia with prednisone, high PPI doses, and budesonide for 6 months [25].

\section{Evidence Suggesting that Achalasia Causes EoE}

Achalasia may cause EoE due to esophageal stasis of retained food material that irritates the mucosa, inducing secretion of chemokines that attract eosinophils. According to this theory, treatments that improve achalasia and esophageal emptying should improve mucosal eosinophilia $[1,3]$. As far as this concept is concerned, there are only a few supporting data documenting the effect of achalasia treatment on esophageal eosinophilia. Mandaliya et al. [26] described achalasia patients with esophageal mucosal biopsies showing eosinophilia of EoE level (i.e., $\geq 15$ eosinophils per hpf). Although histologic response was not adequately assessed and long-term outcome of patients having achalasia with concomitant EoE was quite poor, 4 out of 6 patients presented good short-term response after achalasia treatment [26]. In a report of a patient with concurrent EoE and achalasia, dysphagia, EoE, and motility abnormality did not respond to any corticoid therapy, but dysphagia and EoE improved substantially after pneumatic dilatation of the cardia. The authors supported that eosinophilic infiltration of the esophagus occurred secondary to achalasia in their patient [27]. In a study of 50 achalasia patients who had esophageal mucosal biopsies before and after Heller myotomy, the authors found that 17 patients ( $34 \%$ ) had $\geq 1$ eosinophil per hpf in preoperative biopsies with only one patient having intraepithelial eosinophils $>15 / \mathrm{hpf}$. Out of them, 6 patients (35\%) showed a decrease in esophageal eosinophilia postoperatively. In a proportion of patients, there was a super- imposed candida infection indicating that esophageal eosinophilia in achalasia is likely related to stasis or superimposed fungal infection [28]. The summary of studies revealing a possible relationship between EoE and achalasia is illustrated in Table 1.

\section{Implications and Future Perspectives in Diagnosis and Treatment}

Based on the aforementioned studies, an association between EoE and achalasia may show a possible cause and effect relationship, although it is not clear whether esophageal motility abnormalities that have been described in EoE are the result of EoE or vice versa. The incidence of esophageal motility abnormalities in EoE patients has been reported to be $25-75 \%$ with the majority of dysmotility findings being minor, while EoE may be diagnosed in up to $34 \%$ of achalasia patients [3]. In the study of Weiss AH et al. [29], the occurrence of abnormal esophageal manometry was reported to be between 4 and $87 \%$ among patients with EoE.

Several studies describing presenting symptoms of EoE clearly show that the most common presenting symptom is dysphagia (70-80\%) followed by food impaction $(33-54 \%)[30,31]$. Nearly all achalasia patients also complain of dysphagia and regurgitation $[30,31]$. Therefore, achalasia and EoE may both be suspected in symptomatic patients and concomitant occurrence of both entities should be diagnosed or excluded. Endoscopyesophagogastroduodenoscopy should be always performed in patients with suspected achalasia in order to exclude malignancy of the esophagogastric junction or reveal a concomitant EoE [30]. Inflammatory changes in EoE are frequently patchy and may not be present in all biopsies. It is recommended that at least 6 biopsies should be obtained from at least 2 different locations in the esophagus to increase the diagnostic sensitivity [30]. Esophageal biopsies should be targeted to areas of endoscopic abnormality, mainly white exudates and longitudinal furrows, which are associated with higher peak of eosinophil counts [30]. Biopsies should also be taken despite a normal endoscopic appearance of the esophagus. Random duodenal and gastric mucosal biopsies would help exclude eosinophilic gastroenteritis [30].

The activity of a particular esophageal disease, such as EoE or achalasia, can be evaluated using clinician-reported outcome measures assessing various endoscopic, histologic, functional, and laboratory findings, and patientreported outcome (PRO) measures. The PRO measures 


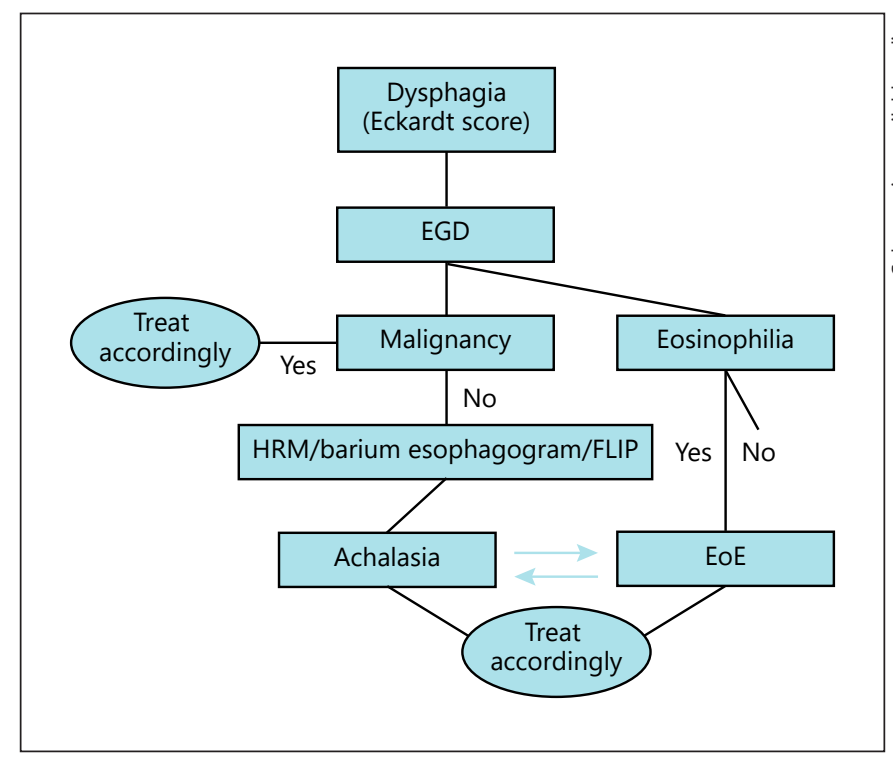

Fig. 1. Diagnostic algorithm of patients with suspected EoE and achalasia. EGD, esophagogastroduodenoscopy; HRM, high-resolution manometry; FLIP, Functional Lumen Imaging Probe; EoE, eosinophilic esophagitis.

are those that are designed to be self-reported by patients. Commonly used PRO instruments include those that assess symptom severity, health-related quality of life, "general" quality of life, or health status [32]. Timed barium esophagogram and Eckardt score may be used in the initial evaluation of patients with simultaneous achalasia and $\mathrm{EoE}$ in order to diagnose and evaluate achalasia severity before and after treatment [31]. Timed barium esophagogram offers an objective evaluation of the disorder, identifying the "bird-beak" appearances of barium and estimating the degree of esophageal emptying with great interobserver agreement, whereas Eckardt score is a simple questionnaire tool measuring symptom severity in achalasia patients focusing on 4 symptom components: dysphagia, regurgitation, retrosternal pain, and weight loss [31]. Eckardt score is the most widely used and accepted questionnaire for achalasia disease severity [31].

High-resolution manometry (HRM) is the test of choice for the diagnosis of achalasia. HRM has been reported to increase the diagnostic accuracy of achalasia compared to conventional manometry [31]. As the incidence of esophageal motility abnormalities in EoE patients is high, HRM is suggested in symptomatic, nonresponders to treatment EoE patients [31]. In the study by Ghisa et al. [23], 41 out of 109 patients with EoE who underwent HRM during initial assessment had esophageal motor disorders. Out of them, 59\% had minor motor dis- orders, and $41 \%$ presented with major motor disorders, including 8 patients with achalasia. Achalasia and nonachalasia obstructive motor disorders had a prevalence of $14.7 \%$ among patients with EoE. In another study using HRM in 24 patients with EoE, Chen et al. [33] found that the most common motility abnormality was elevation in peristaltic velocity; subsets of patients had failed esophageal peristalsis and impaired LES junction relaxation, and 1 patient had a significantly elevated esophageal contractile pressure. In this way, a concurrent esophageal motility abnormality such as achalasia may be diagnosed and a different treatment strategy may be followed.

A new technology that has become available is the Functional Lumen Imaging Probe (FLIP), which could be effectively utilized in assessing the mechanical properties of the esophageal wall and opening dynamics of the esophagogastric junction in various esophageal diseases [34]. FLIP consists of a catheter and an infinitely compliant balloon of varying diameters on the distal end with impedance planimetry electrodes within. It could be placed into the esophagus either transorally or transnasally [34]. FLIP appears to be well suited for the evaluation of patients with achalasia and could be used to provide additive information beyond manometry in the management of achalasia (preoperatively, intraoperatively, and postoperatively) [34]. In EoE patients, FLIP can accurately assess esophageal body narrowing and mechanical properties, and identify the esophageal distensibility plateau, thus making it a significant tool for the assessment of disease severity and clinically relevant outcomes in EoE patients [34]. A proposed diagnostic algorithm is presented in Fig. 1.

As far as treatment options are concerned, conservative methods should probably be utilized initially in patients with concurrent EoE and achalasia, especially in patients without significative esophageal dilation and without radiological barium stasis $>5 \mathrm{~cm}$, whereas more invasive procedures should be kept for resistant cases [30, $35,36]$. Due to low cost, good safety profile, convenience, and a large body of literature describing PPI response in patients with esophageal eosinophilia and endoscopic findings suggestive of EoE, a PPI should be considered as a potential early or initial treatment $[30,35]$. PPI therapy (omeprazole 20-40 mg twice daily or equivalent) is effective in inducing and maintaining EoE remission and improving EoE symptoms [30, 35]. Topical corticosteroids (swallowed puffs, inhalers, or tablets of fluticasone/ budesonide for 2-12 weeks) have been also reported to be effective in inducing and maintaining EoE remission, as well as improving motility abnormalities and achalasia 
symptoms. Recently, an orodispersible tablet of budesonide has been approved as the first "in label" medication for inducement and maintenance of remission in EoE patients [36]. Systemic steroids are not recommended due to systemic effects (hyperphagia, weight gain, and/ or cushingoid features) $[20,30,36]$.

Since EoE is an allergic immune-mediated chronic disease of the esophagus and various food diets can trigger EoE, various dietary elimination therapies have been used as alternatives to chronic medications [30]. Elimination diets have multiple benefits in EoE patients; they prevent the need for chronic medications, may reduce systemic inflammation in EoE, and can allow diagnosing food triggers in EoE through the process of sequential food reintroductions [30]. On the other hand, elimination and elemental diet do not seem to improve achalasia symptoms and, therefore, are not recommended in the management of EoE and achalasia patients [30]. Endoscopic dilation (ED) is a safe procedure that improves dysphagia and achalasia symptoms in up to 3 quarters of EoE and achalasia patients without having an effect on the underlying esophageal inflammation and should be considered in achalasia patients with dysphagia/food impaction unresponsive to conservative treatment [30].

In EoE and achalasia patients with persistent symptoms after conservative treatment has failed, more invasive procedures to improve achalasia symptoms may be used. Laparoscopic Heller myotomy with or without partial fundoplication or POEM has been reported to be the most effective in eliminating achalasia symptoms [17, 28]. Botulinum toxin injection should be reserved for patients who are unfit for surgery or as a bridge to more effective therapies, such as surgery or ED, although promising results have been reported in patients with EoE and achalasia [31]. In patients with end-stage achalasia and EoE, esophagectomy may be indicated after failure of previous conservative or less invasive treatments (ED, POEM, and laparoscopic Heller myotomy) [31].

Following treatment of EoE, upper gastrointestinal endoscopy with esophageal biopsies should be performed to examine the therapeutic outcome after a 6- to 12-week interval [28]. Timed barium esophagogram and Eckardt score may also be used in the follow-up assessment in patients with simultaneous achalasia and EOE to evaluate the therapeutic outcome [31].

\section{Conclusion}

In conclusion, a possible association seems to exist between EoE and achalasia although current literature cannot determine which is the primary condition and which is the result. Physicians should be suspicious of a possible coexistence between EoE and achalasia and diagnostic exams should target in detection and diagnosis of both conditions. The concept that eosinophils in esophageal muscle might underlie motility disorders has important therapeutic implications. It suggests that some motility disorders like achalasia might respond to treatments that decrease muscular infiltration by eosinophils, whereas the medications commonly used to treat EoE might not affect the eosinophil-induced motility abnormalities in patients with esophageal mucosa eosinophilia. All in all, some patients with both EoE and achalasia respond well and benefit from conservative treatments, while more invasive procedures should probably be reserved for more complex and resistant cases. However, in order to reach solid conclusions, larger multicenter studies are needed.

\section{Conflict of Interest Statement}

All the authors declare that they have no potential conflict of interest.

\section{Funding Sources}

None of the authors have received any financial funding from any source. No funding was granted for this article.

\section{Author Contributions}

Dimitrios Schizas: study conception, drafting of the manuscript, analysis and interpretation of data, and critical revision of the manuscript; Athanasios Syllaios: acquisition of data, drafting of the manuscript, and analysis and interpretation of the data; $\mathrm{Mi}$ chail Vailas: drafting of the manuscript and analysis and interpretation of the data; Maria Sotiropoulou: drafting of the manuscript and analysis and interpretation of the data; Tania Triantafyllou: analysis and interpretation of the data, drafting of the manuscript; Dimitrios Tsapralis: analysis and interpretation of the data, drafting of the manuscript; Ioannis S. Papanikolaou: analysis and interpretation of the data, drafting of the manuscript; Dimitrios Theodorou: study conception and design, acquisition of data, and critical revision of the manuscript. 


\section{References}

1 Spechler SJ. Eosinophilic esophagitis: novel concepts regarding pathogenesis and clinical manifestations. J Gastroenterol. 2019;54(10): 837-44.

2 Velikova T. Challenges and updates in the management of eosinophilic esophagitis. Prz Gastroenterol. 2020;15(1):27-33.

3 Spechler SJ, Konda V, Souza R. Can eosinophilic esophagitis cause achalasia and other esophageal motility disorders?. Am J Gastroenterol. 2018 Nov;113(11):1594-9.

4 de Bortoli N, Penagini R, Savarino E, Marchi S. Eosinophilic esophagitis: update in diagnosis and management. Position paper by the Italian Society of Gastroenterology and Gastrointestinal Endoscopy (SIGE). Dig Liver Dis. 2017 Mar;49(3):254-60.

5 Papadopoulou A, Koletzko S, Heuschkel R, Dias JA, Allen KJ, Murch SH, et al. ESPGHAN eosinophilic esophagitis working group and the gastroenterology committee. Management guidelines of eosinophilic esophagitis in childhood. J Pediatr Gastroenterol Nutr. 2014 Jan;58(1):107-18.

6 Oude Nijhuis RAB, Zaninotto G, Roman S, Boeckxstaens GE, Fockens P, Langendam MW, et al. European guidelines on achalasia: united European gastroenterology and European society of neurogastroenterology and motility recommendations. United Eur Gastroenterol J. 2020 Feb;8(1):13-33.

7 Hirano I, Chan ES, Rank MA, Sharaf RN, Stollman NH, Stukus DR, et al. AGA Institute clinical guidelines committee; Joint task force on allergy-immunology practice parameters. AGA Institute and the joint task force on allergy-immunology practice parameters clinical guidelines for the management of eosinophilic esophagitis. Gastroenterology. 2020 May;158(6):1776-86.

8 Ates F, Vaezi MF. The pathogenesis and management of achalasia: current status and future directions. Gut Liver. 2015;9(4):449-63.

9 Pandolfino JE, Gawron AJ. Achalasia: a systematic review. JAMA. 2015;313(18):184152.

10 Nurko S, Rosen R. Esophageal dysmotility in patients who have eosinophilic esophagitis. Gastrointest Endosc Clin N Am. 2008;18(1): 73-89.

11 Nelson MR, Zhang X, Pan Z, Spechler SJ, Souza RF. Mast cell effects on esophageal smooth muscle and their potential role in eosinophilic esophagitis and achalasia. Am J Physiol Gastrointest Liver Physiol. 2020 Dec 23;320: G319-27.

12 Landres RT, Kuster GG, Strum WB. Eosinophilic esophagitis in a patient with vigorous achalasia. Gastroenterology. 1978;74(6): 1298-301.
13 Goldblum JR, Whyte RI, Orringer MB, Appelman HD. Achalasia. A morphologic study of 42 resected specimens. Am J Surg Pathol. 1994;18(4):327-37.

14 Goldblum JR, Rice TW, Richter JE. Histopathologic features in esophagomyotomy specimens from patients with achalasia. Gastroenterology. 1996;111(3):648-54.

15 Sodikof JB, Lo AA, Shetuni BB, Kahrilas PJ, Yang GY, Pandolfino JE, et al. Histopathologic patterns among achalasia subtypes. Neurogastroenterol Motil. 2016;28:139-45.

16 Tøttrup A, Fredens K, Funch-Jensen P, Aggestrup S, Dahl R. Eosinophil infiltration in primary esophageal achalasia. A possible pathogenic role. Dig Dis Sci. 1989;34(12): 1894-9.

17 Jin H, Wang B, Zhang LL, Zhao W. Activated eosinophils are present in esophageal muscle in patients with achalasia of the esophagus. Med Sci Monit. 2018;24:2377-83.

18 Hejazi RA, Reddymasu SC, Sostarich S, McCallum RW. Disturbances of esophageal motility in eosinophilic esophagitis: a case series. Dysphagia. 2010;25(3):231-7.

19 Nakajima N, Sato H, Takahashi K, Hasegawa G, Mizuno K, Hashimoto S, et al. Muscle layer histopathology and manometry pattern of primary esophageal motility disorders including achalasia. Neurogastroenterol Motil. 2017;29(3):e12968

20 Nennstiel S, Bajbouj M, Becker V, Slotta-Huspenina J, Wagenpfeil S, Schmid RM, et al. High-resolution manometry in patients with eosinophilic esophagitis under topical steroid therapy-a prospective observational study (HIMEOS-study). Neurogastroenterol Motil. 2016;28:599-607.

21 Lucendo AJ, Castillo P, Martín-Chávarri S, Carrión G, Pajares R, Pascual JM, et al. Manometric findings in adult eosinophilic oesophagitis: a study of 12 cases. Eur J Gastroenterol Hepatol. 2007;19(5):417-24.

22 Savarino E, Gemignani L, Zentilin P, De Bortoli N, Malesci A, Mastracci L, et al. Achalasia with dense eosinophilic infiltrate responds to steroid therapy. Clin Gastroenterol Hepatol. 2011;9(12):1104-6.

23 Ghisa M, Laserra G, Marabotto E, Ziola S, Tolone S, de Bortoli N, et al. Achalasia and obstructive motor disorders are not uncommon in patients with eosinophilic esophagitis. Clin Gastroenterol Hepatol. 2020;S1542-3565(20): 31066-1.

24 Mathur A, Mundada K, Verma A, Agrawal V, Verma V, Ghoshal UC. Eosinophilic gastroenteritis presenting as achalasia cardia and responding to botulinum injection and therapy directed against eosinophilic esophagitis. Indian J Gastroenterol. 2019 Dec;38(6):554-6.
25 Surdea-Blaga T, David L, Botan EC, Dumitrascu DL. Achalasia-like changes in eosinophilic esophagitis. J Gastrointestin Liver Dis. 2019 Jun 1;28:146.

26 Mandaliya R, DiMarino AJ, Cohen S. Association of achalasia and eosinophilic esophagitis. Indian J Gastroenterol. 2013;32(1):54-7.

27 Frieling T, Heise J, Kreysel C, Blank M, Hemmerlein B, Beccu L, et al. Eosinophilic esophagitis and achalasia - just a coincidence?. Z Gastroenterol. 2019 Feb;57(2):151-5.

28 Cools-Lartigue J, Chang SY, Mckendy K, Mayrand S, Marcus V, Fried GM, et al. Pattern of esophageal eosinophilic infiltration in patients with achalasia and response to Heller myotomy and Dor fundoplication. Dis Esophagus. 2013;26(8):766-75.

29 Weiss AH, Iorio N, Schey R. Esophageal motility in eosinophilic esophagitis. Rev Gastroenterol Mex. 2015 Jul-Sep;80(3):205-13.

30 Lucendo AJ, Molina-Infante J, Arias Á, von Arnim U, Bredenoord AJ, Bussmann C, et al. Guidelines on eosinophilic esophagitis: evidence-based statements and recommendations for diagnosis and management in children and adults. United Eur Gastroenterol J. 2017;5(3):335-58.

31 Zaninotto G, Bennett C, Boeckxstaens G, Costantini M, Ferguson MK, Pandolfino JE, et al. The 2018 ISDE achalasia guidelines. Dis Esophagus. 2018 Sep 1;31(9).

32 Schoepfer A, Straumann A, Safroneeva E. Patient-reported outcomes in eosinophilic esophagitis and achalasia. Curr Treat Options Gastroenterol. 2016 Mar;14(1):51-60.

33 Chen J, Ghosh S, Pandolfino J, Kharilas PJ, Hirano I. Esophageal dysmotility in eosinophilic esophagitis: analysis using high resolution esophageal manometry. Gastroenterology. 2007;132:A-6.

34 Hirano I, Pandolfino JE, Boeckxstaens GE. Functional lumen imaging probe for the management of esophageal disorders: expert review from the clinical practice updates committee of the AGA Institute. Clin Gastroenterol Hepatol. 2017 Mar;15(3):325-34.

35 Dellon ES, Liacouras CA, Molina-Infante J, Furuta GT, Spergel JM, Zevit N, et al. Updated international consensus diagnostic criteria for eosinophilic esophagitis: proceedings of the AGREE conference. Gastroenterology. 2018;155(4):1022-e10.

36 Hao LX, Lu Y, Li T, Gong B. A meta-analysis of efficacy of topical steroids in eosinophilic esophagitis: from the perspective of histologic, clinical, and endoscopic outcome. Gastroenterol Hepatol. 2020 Oct 2120;44:251-60. 